\title{
Current Insights on the Treatment of Anaplastic Lymphoma Kinase-Positive Metastatic Non-Small Cell Lung Cancer: Focus on Brigatinib
}

\author{
Erika Rijavec $\mathbb{D}^{1}$, Federica Biello ${ }^{2}$, Alice Indini ${ }^{3}$, Francesco Grossi ${ }^{3}$, Carlo Genova $\mathbb{D}^{4,5}$ \\ 'Medical Oncology Unit, Fondazione IRCCS Ca' Granda Ospedale Maggiore Policlinico, Milano, Italy; ${ }^{2}$ Division of Oncology, Department of \\ Translational Medicine, University of Piemonte Orientale, Novara, Italy; ${ }^{3}$ Division of Medical Oncology, University of Insubria, ASST dei Sette Laghi, \\ Varese, Italy; ${ }^{4} \mathrm{UO}$ Clinica di Oncologia Medica, IRCCS Ospedale Policlinico San Martino, Genova, Italy; ${ }^{5}$ Department of Internal Medicine and Medical \\ Specialties (DIMI), Università degli Studi di Genova, Genova, Italy \\ Correspondence: Erika Rijavec, Email ery80x@yahoo.it
}

\begin{abstract}
Rearrangement of anaplastic lymphoma kinase $(A L K)$ gene is detected in approximately $5 \%$ of non-small cell lung cancer (NSCLC) patients. Tyrosine kinase inhibitors targeting ALK have significantly improved the prognosis of these patients. However, most patients experienced disease progression within a few years due to acquired resistance. Brigatinib is a second-generation ALK inhibitor effective in presence of several $A L K$ mutations with demonstrated activity against central nervous system metastases. Currently, brigatinib is approved to treat ALK-positive metastatic NSCLC patients not previously treated with ALK inhibitors and patients who have progressed on or are intolerant to crizotinib. In this review, we provide a summary of results from clinical trials involving brigatinib, and we discuss its possible role in the management of ALK-positive NSCLC in the following years.
\end{abstract}

Keywords: brigatinib, non-small cell lung cancer, anaplastic lymphoma kinase

\section{Introduction}

Lung cancer is the second most frequently diagnosed cancer worldwide, after breast cancer, with an estimated 2.2 million new cases in 2020, and its incidence continues to rise. An estimated 1.8 million lung cancer deaths occurred in 2020. Among all malignancies, lung cancer is currently the leading cause of cancer deaths in several countries, especially in Europe and North America. ${ }^{1}$

In the last decades, the landscape of non-small cell lung cancer (NSCLC) treatment has drastically evolved thanks to the approval of immune checkpoint inhibitors (ICIs) and, for patients with targetable driver mutations, the advent of several tyrosine kinase inhibitors that improved survival, disease and symptom control, and quality of life.

In NSCLC, a small inversion within chromosome $2 p$ short arm results in the formation of a fusion gene comprising portions of the anaplastic lymphoma kinase $(A L K)$ gene and the echinoderm microtubule-associated protein-like 4 $(E M L 4)$ gene. This rearrangement produces a fusion protein with constitutive kinase activity and transforming capacity. ${ }^{2}$ $A L K$ rearrangements are detected in approximately $4-5 \%$ of all NSCLC, especially in patients with lung adenocarcinoma, under 50 years of age, and never or light ex-smokers. ${ }^{3-5}$

The presence of $A L K$ rearrangements makes the tumor sensitive to tyrosine kinase inhibitors, which bind to receptor tyrosine kinase domain, resulting in inhibition of the downstream signaling pathways. ${ }^{6}$

Crizotinib was the first ALK inhibitor to be approved by the US Food and Drug Administration (FDA) for the treatment of advanced ALK-positive NSCLC tumors, with fluorescence in situ hybridization (FISH) assay (Vysis ${ }^{\circledR}$ ALK Break-Apart FISH Probe Kit test; Abbott Molecular) as the recommended diagnostic test for detection. ${ }^{7}$

Then, in the attempt to overcome crizotinib-acquired resistance, frequently due to secondary mutations in the $A L K$ gene (such as C1156Y, L1196M, F1174L, and F1174V), the second generation of ALK inhibitors (ceritinib, alectinib, and 
brigatinib) have been developed. More recently, novel second- and third-generation ALK inhibitors (entrectinib, ensartinib, and lorlatinib) have been developed to overcome resistance in ALK-positive NSCLC patients. ${ }^{6}$

The aim of this review is to provide an overview of the current state of the art and most recent evidence from randomized clinical trials evaluating brigatinib to discuss the role of this ALK inhibitor in treating ALK-positive NSCLC patients.

\section{Brigatinib: Pharmacodynamic and Pharmacokinetics Properties}

Brigatinib is a second-generation tyrosine kinase inhibitor of ALK, ROS1, and insulin-like growth factor-1 receptor (IGF1R), with activity against EGFR deletions and mutations, including T790M. Brigatinib is about $90 \%$ bound to human plasma proteins, and its mean elimination half-life is $25 \mathrm{~h}$. Brigatinib is primarily metabolized by CYP2C 8 and CYP3A in vitro. ${ }^{8}$ Results from a multi-arm, drug-drug interaction (DDI) study showed that strong CYP2C8 inhibitors might be administered without any brigatinib dose adjustments because they do not have a clinically meaningful effect on brigatinib pharmacokinetics. Instead, the coadministration of strong CYP3A inhibitors should be avoided since they increase the systemic exposure of brigatinib. ${ }^{9}$ Brigatinib can be administered with or without food because the consumption of a high-fat meal does not have a meaningful effect on the drug's pharmacokinetics. ${ }^{10}$

Interestingly, brigatinib exhibits in vitro activity against a panel of 17 ALK mutants associated with resistance to crizotinib, ceritinib, and alectinib. Brigatinib had 2.2- to 77-fold more potency than crizotinib against all but the L1198F mutant, was >3-fold more potent than alectinib against L1152R, I1171N, V1180L, L1196M, G1202R, and G1269, and $>3$-fold more potent than ceritinib against L1152R/P, C1156Y, L1198F, and G1269A. ${ }^{11}$ Notably, brigatinib demonstrated a higher activity than lorlatinib for the G1269A mutation. ${ }^{12}$

\section{Clinical Trials}

Several clinical trials investigated the administration of brigatinib for the treatment of advanced ALK-positive NSCLC. Results are summarized in Table 1.

\section{Phase I/2 and ALTA Trial}

The safety and the activity of brigatinib were investigated in 137 patients affected by advanced solid tumors, including 79 NSCLC patients harboring $A L K$ gene rearrangements, in a phase I/II study.

The primary endpoint of the Phase I dose-escalation was to establish the recommended Phase II dose. The primary endpoint of the expansion phase II was the objective response, defined as the proportion of patients with complete or partial response. In the expansion phase, three oral once-daily regimens: $180 \mathrm{mg}, 90 \mathrm{mg}$, and $180 \mathrm{mg}$ with a 7-day lead-in at $90 \mathrm{mg}$ have been assessed in five cohorts: ALK inhibitor-naive ALK-positive NSCLC (cohort 1), crizotinib-treated ALK-positive NSCLC (cohort 2), EGFR T790M-positive NSCLC progressed after one previous EGFR tyrosine kinase inhibitor (cohort 3), other tumors with abnormalities in ALK or ROS1 (cohort 4), and ALK-positive NSCLC with active and measurable brain metastases (cohort 5). Notably, pulmonary events with early-onset (EOPEs) were described in 11 (8\%) patients. They occurred during the first seven days of treatment, generally within 24-48 hours. These events, including dyspnea, cough, hypoxia, pneumonia, and pneumonitis, are often associated with chest imaging findings such as ground-glass opacities or diffuse alveolar damage on histopathology, in the absence of a clear alternative cause.

Two patients with EOPEs died, and six (55\%) patients did not resume brigatinib after pulmonary toxicity. EOPEs were more frequently observed in patients treated with brigatinib at the dose of $180 \mathrm{mg}$ daily. Due to this finding, the recommended phase II doses were: $90 \mathrm{mg}$ daily and $180 \mathrm{mg}$ daily with a 7-day lead-in at $90 \mathrm{mg}$.

Efficacy data of brigatinib were encouraging: a confirmed objective response was detected in four of four $(100 \%)$ patients enrolled in cohort 1, in 31 of $42(74 \%)$ patients in cohort 2, in none of one patient $(0 \%)$ in cohort 3, in three of 18 $(17 \%)$ patients in cohort 4 , and in five of six $(83 \%)$ patients in cohort 5 . Although the small number of patients with brain metastases enrolled in this trial, brigatinib showed a promising intracranial activity at the dose of $90 \mathrm{mg}$ daily or greater. Furthermore, median progression-free survival (PFS) was not reached in crizotinib-naive ALK-positive NSCLC and was 13.2 months in ALK-positive NSCLC pre-treated with crizotinib. Brigatinib showed an acceptable safety profile: the 
Table I Clinical Trials with Brigatinib in Advanced ALK-Positive NSCLC

\begin{tabular}{|c|c|c|c|c|c|c|}
\hline & Phase & Patients & Key Inclusion Criteria & Treatment & $\begin{array}{l}\text { Primary } \\
\text { Endpoint }\end{array}$ & Results \\
\hline NCT0 I 44946 I & $1 / I I$ & 137 & $\begin{array}{l}\text { No previous ALK-targeted TKIs, with the } \\
\text { exception of crizotinib. }\end{array}$ & $\begin{array}{l}\text { Phase I: brigatinib } 30 \\
-300 \mathrm{mg} \\
\text { Phase II: brigatinib } 90 \mathrm{mg} \text {; } \\
\text { or } 180 \mathrm{mg} \text { or } 180 \mathrm{mg} \\
\text { with a 7-day lead-in at } 90 \\
\mathrm{mg}\end{array}$ & $\begin{array}{c}\text { ORR } \\
\text { (phase II) }\end{array}$ & $\begin{array}{l}\text { Cohort I: } \\
\text { ORR: I00\% } \\
\text { Cohort 2: } \\
\text { ORR: 74\% } \\
\text { Cohort 3: } \\
\text { ORR: 0\% } \\
\text { Cohort 4: } \\
\text { ORR: I7\% } \\
\text { Cohort 5: } \\
\text { ORR: } 83 \%\end{array}$ \\
\hline $\begin{array}{l}\text { NCT02094573 } \\
\text { ALTA }\end{array}$ & II & 222 & $\begin{array}{l}\text { Locally advanced or metastatic ALK+ } \\
\text { NSCLC. } \\
\text { Progressive disease while on crizotinib. }\end{array}$ & $\begin{array}{l}\text { Arm A: brigatinib } 90 \mathrm{mg} \\
\text { Arm B: brigatinib } 180 \mathrm{mg} \\
\text { with a 7-day lead-in at } 90 \\
\mathrm{mg}\end{array}$ & ORR & $\begin{array}{c}\text { Arm A: ORR: } \\
46 \% \\
\text { Arm B: ORR: } \\
56 \%\end{array}$ \\
\hline $\begin{array}{l}\text { NCT0273750I } \\
\text { ALTA-IL }\end{array}$ & III & 275 & $\begin{array}{l}\text { Stage IIIB (locally advanced or recurrent } \\
\text { and not a candidate for definitive } \\
\text { multimodality therapy) or stage IV ALK+ } \\
\text { NSCLC. } \\
\text { No previous ALK-targeted TKIs. } \\
\text { No more than one previous systemic } \\
\text { treatment for advanced NSCLC }\end{array}$ & $\begin{array}{l}\text { Brigatinib } 180 \mathrm{mg} \text { with a } \\
\text { 7-day lead-in at } 90 \mathrm{mg} \\
\text { or } \\
\text { crizotinib, } 250 \mathrm{mg} \text { twice } \\
\text { daily }\end{array}$ & $\begin{array}{c}\text { BIRC- } \\
\text { assessed PFS }\end{array}$ & $\begin{array}{l}\text { 3-year BIRC } \\
\text { PFS: } 43 \% \\
\text { (brigatinib) } \\
19 \% \\
\text { (crizotinib) }\end{array}$ \\
\hline $\begin{array}{l}\text { NCT034I0I08 } \\
\text { J-ALTA }\end{array}$ & II & 104 & $\begin{array}{l}\text { Brigatinib } 180 \mathrm{mg} \text { with a 7-day lead-in at } \\
90 \mathrm{mg}\end{array}$ & $\begin{array}{l}\text { Stage IIIB/IIIC/IV ALK- } \\
\text { positive NSCLC. } \\
\text { No previous ALK- } \\
\text { targeted TKIs. }\end{array}$ & $\begin{array}{l}\text { ORR (main } \\
\text { refractory } \\
\text { expansion } \\
\text { cohort)* }\end{array}$ & ORR: $34 \%$ \\
\hline
\end{tabular}

Notes: *Independent review committee (IRC)-assessed confirmed ORR.

Abbreviations: TKIs, tyrosine kinase inhibitors; NSCLC, non-small cell lung cancer; ORR, objective response rate; PFS, progression-free survival; BIRC, blinded independent central review.

most common adverse events (AEs) described were nausea, diarrhea, and fatigue, while the most severe (grade 3 or 4) AEs reported included elevated lipase, hypertension, and dyspnea. ${ }^{13}$

Kim et al, in the randomized phase II ALK in Lung Cancer Trial of AP26113/brigatinib (ALTA), evaluated brigatinib in 222 patients affected by locally advanced or metastatic ALK-positive NSCLC who had progressed on crizotinib. All patients had NSCLC with a documented $A L K$ rearrangement based on an FDA-approved test or a different test with adequate archival tissue to confirm $A L K$ arrangement by the Vysis ${ }^{\circledR}$ ALK Break-Apart FISH Probe Kit test. Patients, stratified by intracranial metastases and best response to crizotinib, were randomized (1:1) to receive brigatinib $90 \mathrm{mg}$ daily (arm A) or $180 \mathrm{mg}$ daily with a lead-in (arm B). Crossover to arm B was allowed in patients treated with brigatinib at $90 \mathrm{mg}$ daily after progression. The primary endpoint of investigator-assessed confirmed objective response rate (ORR) was $45 \%$ and $54 \%$ in arms $\mathrm{A}$ and $\mathrm{B}$, respectively. In patients with measurable baseline intracranial metastasis, the independent review committee (IRC)-assessed intracranial ORRs seen were $42 \%$ and $67 \%$ in arms A and B, respectively. The most common AEs $(>25 \%)$ included nausea, diarrhea, headache, and cough. The most frequent grade $\geq 3$ AEs described were hypertension, increased blood creatine phosphokinase (CPK), pneumonia, and increased lipase. Fourteen patients treated with brigatinib $90 \mathrm{mg}$ daily experienced EOPEs. In six of 14 patients, after dose interruption, the administration of brigatinib was resumed. Results from a multivariable analysis demonstrated that patients with older age had an increased risk of developing EOPEs. Fatal adverse reactions have been documented in $3.7 \%$ of patients and consisted of pneumonia, dyspnea, respiratory failure, sudden death, pulmonary embolism, bacterial meningitis, and urosepsis. $^{14}$ 
In April 2017, based on the ALTA trial results, the FDA granted accelerated approval for brigatinib, at the recommended dose of $180 \mathrm{mg}$ daily with a 7-day lead-in at $90 \mathrm{mg}$, for the treatment of stage IV, ALK-positive, NSCLC patients, who have progressed on or are intolerant to crizotinib. ${ }^{15}$

Camidge et al performed a comprehensive exploratory analysis of the efficacy and safety of brigatinib in patients with ALK-rearranged NSCLC, with baseline brain metastases, enrolled in the phase I/II study and in the ALTA study. As determined by IRC, a total of 50 patients in the phase 1/2 study and 153 patients in the ALTA study were included in this analysis. IRC-assessed confirmed intracranial ORRs were as follows: ORR was seen in 8/15 patients (53\%) in the phase $1 / 2$ study, in 12/18 patients (67\%) and 12/26 patients (46\%) in ALTA arm A and B, respectively. This analysis showed that in the phase I/II study confirmed intracranial ORRs were similar or better in patients without prior radiation therapy, and in the ALTA trial ORRs were similar or better in patients with active, measurable brain metastases.

Median intracranial PFS was 14.6 months in the phase $1 / 2$ study, 15.6 and 18.4 months in ALTA arm A and B, respectively. ${ }^{16}$

Huber et al performed an updated survival and efficacy analysis of the phase II ALTA trial after approximately two years of follow-up. Consistent with previously reported results, brigatinib (180 $\mathrm{mg}$ daily with a lead-in) continued to demonstrate to be a very attractive option for ALK-positive NSCLC patients who progressed on crizotinib.

The investigator-assessed confirmed ORR was $46 \%$ in arm A, and 56\% in arm B. Median IRC-assessed PFS and median overall survival (OS) were improved with the higher dose of brigatinib (16.7 months vs 9.2 months and 34.1 months vs 25.9 months, respectively). ${ }^{17}$ Notably, the median IRC-assessed PFS achieved with brigatinib was longer than PFS reported with other second- and third-generation ALK inhibitors in the same setting. ${ }^{18-20}$ Brigatinib confirmed a marked intracranial anti-tumor activity: IRC-confirmed intracranial ORR in patients with measurable brain lesions at baseline was seen in 13/26 patients (50\%) in arm A and in 12/18 patients (67\%) in arm B. In arms A and B, IRC-assessed intracranial PFS was 12.8 months and 18.4 months, respectively. After a longer follow-up, the authors reported no new safety findings. ${ }^{17}$

Patients reported outcomes (PROs) were collected in patients enrolled in the ALTA study by using the European Organization for Research and Treatment of Cancer (EORTC) Quality of Life Questionnaire - Core 30 (QLQ-C30) at baseline, on the first day of each cycle, at treatment discontinuation, and at end-of-treatment (EOT). The administration of brigatinib improved Global Health Status (GHS)/QOL scores and reduced pain and dyspnea scores. ${ }^{21}$ Kawata et al evaluated the impact of brigatinib treatment on health utilities mapping QLQ-C30 scores to European Quality-of-Life-5 Dimensions (EQ-5D) utility scores thanks to two published algorithms. This exploratory analysis showed that both algorithms suggested that brigatinib improved health utility in ALK-positive NSCLC patients who progressed on crizotinib. $^{22}$

During the 2021 American Society of Clinical Oncology (ASCO) Annual Meeting, Gettinger presented phase I/II study and Phase 2 ALTA trial final results.

At a median follow-up of 27.7 months, in the phase 1/2 study, brigatinib showed in ALK-positive NSCLC patients a confirmed investigator-assessed ORR of $67 \%$ with a median duration of response (DOR) of 14.9 months. The median investigator-assessed PFS was 14.5 months, and median OS was 47.6 months. The estimated 5-year OS rate was $42 \%$. At the end of the study, four patients were still on treatment.

In the phase II ALTA study, brigatinib at the approved dosing regimen continued to demonstrate superior efficacy and survival benefit with 28.3 months of follow-up. In arms A and B, confirmed investigator-assessed ORR was 51\% and $63 \%$, respectively. Median investigator-assessed and IRC-assessed PFS were 9.2 months and 9.9 months in arm A and 15.6 months and 16.7 months, respectively, in arm B. Median OS was significantly prolonged in arm A compared to arm B (25.9 months vs 40.6 months). The estimated 5-year OS rate was $31 \%$ and $43 \%$ in arms A and B, respectively. At the end of the study, ten patients were still on treatment. ${ }^{23}$

\section{ALTA- I L Trial}

In the multicenter, international, randomized, Phase III, ALTA-1L study, brigatinib (at the dose of $180 \mathrm{mg}$ daily with a 7day lead-in at $90 \mathrm{mg}$ ) was compared to crizotinib (250 mg twice daily). A total of 275 patients affected by locally advanced or metastatic ALK-rearranged NSCLC, not previously treated with ALK inhibitors, were randomized (1:1) 
between the two groups. Crossover to brigatinib was allowed in the crizotinib arm after by blinded independent central review (BIRC)-assessed progression. The primary endpoint of the study was PFS assessed by BICR. At the first interim analysis, the median PFS was 11 months in the brigatinib arm and 9.3 months in the crizotinib arm. The estimated PFS rate at 12 months was significantly higher with brigatinib than crizotinib ( $67 \%$ vs $43 \%$; HR, $0.49, p<0.001$ ). Confirmed ORRs and intracranial response rates were higher in patients treated with brigatinib than patients who received crizotinib ( $71 \%$ vs $60 \%$ and $78 \%$ vs $29 \%$, respectively).

The most common any-grade AEs observed with brigatinib were diarrhea (49\%), increased CPK (39\%), cough (25\%), hypertension (23\%), nausea (26\%), vomiting (18\%), and increased aminotransferases (19\%) and lipase (19\%). Any-grade EOPEs occurred in 3\% of patients in the brigatinib arm and no patients in the crizotinib arm.

Grade $\geq 3$ AEs occurred in $61 \%$ of the brigatinib arm and $55 \%$ of the crizotinib arm. ${ }^{24}$

An exploratory analysis investigating the association of oncogenic alterations including $A L K$ fusion variants and TP53 status, and clinical outcomes has been performed.

Brigatinib showed greater activity than crizotinib in NSCLC patients with poor prognosis, such as those with EML4$A L K$ fusion variant 3 and TP53 mutation. ${ }^{25-28}$

Results of the second interim analysis of the ALTA-1L trial confirmed the superior efficacy, especially among patients with brain metastases at baseline, of brigatinib over crizotinib.

At a median follow-up of 24.9 months, BIRC-assessed PFS was significantly longer in patients treated with brigatinib than those who received crizotinib: 24 months vs 11 months (HR, 0.49; $<<0.0001$ ). No new safety concerns have been reported. ${ }^{29}$

Furthermore, brigatinib was the first ALK inhibitor to significantly improve health-related quality of life (HRQOL) compared with crizotinib in the first-line setting. These results could be explained by the greater efficacy of brigatinib and the fewer clinically relevant side effects associated with the treatment. HRQOL was assessed using EORTC QLQ-C30 and lung cancer-specific module (QLQ-LC13). ${ }^{30}$

Based on the ALTA-1L study, in May 2020, the FDA approved brigatinib for the first-line treatment of metastatic ALK-positive NSCLC and the Vysis ALK Break Apart FISH Probe Kit as a companion diagnostic for brigatinib. ${ }^{31}$

Recently, Camidge et al reported the final results of the ALTA-1L study, confirming what was observed with the first two interim analyses. The 3-year BIRC-assessed PFS was 43\% with brigatinib and 19\% with crizotinib (HR, 0.48). In patients with brain metastasis at baseline, brigatinib significantly prolonged BIRC-assessed intracranial PFS compared to crizotinib (24 months vs 5.5 months). In this subset of patients, the risk of intracranial progression was reduced by $71 \%$ (HR, 0.29) with brigatinib compared with crizotinib. Although OS data were still maturing, posthoc analyses suggested, in patients with baseline brain metastases, an OS benefit for brigatinib (HR, 0.43). ${ }^{32}$

\section{J-ALTA Trial}

The safety and activity of brigatinib (180 mg once daily with a 7-d lead-in at $90 \mathrm{mg}$ once daily) have been explored in the multi-cohort, Phase II, J-ALTA trial enrolling Japanese patients affected by stage IIIB/IIIC/IV ALK-positive NSCLC. The study was composed of a safety lead-in part 1, followed by a refractory expansion part 2 with two cohorts of patients refractory to ALK inhibitors, and part 3 enrolling ALK inhibitors-naïve patients.

The primary endpoint of the main refractory expansion cohort was IRC-assessed confirmed ORR.

A total of 9 patients previously treated with no more than three regimens of systemic anticancer therapy, other than ALK inhibitors, have been included in part 1.

One patient experienced one dose-limiting toxicity (DLT) event of asymptomatic grade 3 lipase increase leading to a brigatinib interruption for 15 days. Therefore, the standard dose of brigatinib $180 \mathrm{mg}$ once daily (with a 7-d lead-in at 90 $\mathrm{mg}$ ) was recommended in part 2.

Part 2 of the study consisted of two cohorts: a main cohort, enrolling 47 patients who progressed on alectinib or alectinib after crizotinib, and an exploratory cohort enrolling 16 patients previously treated with other combinations of up to two previous ALK inhibitors.

The study demonstrated the efficacy of brigatinib in the subset of patients with alectinib-refractory advanced ALKpositive NSCLC. 
Indeed, at the updated analysis cutoff (January 22, 2020), in the main cohort, IRC-assessed ORR and disease control rate (DCR) was $34 \%$ and $79 \%$, respectively. Among eight patients with measurable brain lesions at baseline, the confirmed intracranial ORR was $25 \%$.

Among all 72 patients refractory to ALK inhibitors, three had the G1202R mutation (one of them experienced an IRC-assessed confirmed objective response), and 11 patients had other secondary mutations (six of them had IRCassessed confirmed objective response).

Brigatinib showed a manageable safety profile: the most common AEs (observed in more than $25 \%$ of patients) were elevated serum levels of CPK, lipase, amylase, and aspartate aminotransferase, and diarrhea, hypertension, nausea, and stomatitis. $^{33}$

During the 2021 ASCO Annual Meeting, Kondo presented the primary analysis results of J-ALTA study part 3, enrolling 32 patients who had not previously been treated with an ALK inhibitor. The primary endpoint, 12-month IRCassessed PFS, was 93\%, and IRC-assessed ORR was 97\%. The most frequent AEs reported were increased CPK, hypertension, and diarrhea. Three cases of interstitial lung disease/pneumonitis were described in arm 3; all were grade 1 and occurred after day 15 of treatment. ${ }^{34}$

\section{ALTA-2 Trial}

The ALTA-2 study is a phase II trial investigating brigatinib in locally advanced or metastatic ALK-positive NSCLC patients who have progressed on second-generation ALK inhibitors (alectinib or ceritinib). Patients were enrolled at sites in North America, Europe, and the Asia-Pacific region.

The primary endpoint of the study is IRC-assessed confirmed ORR. The study has completed the enrollment of 104 patients, and results for the primary endpoint may be published shortly. ${ }^{35}$

\section{Clinical Trials Ongoing}

Currently, several clinical trials investigating brigatinib in ALK-positive NSCLC are enrolling.

The currently ongoing Phase II-III clinical trials are summarized in Table 2.

The ABP trial is a randomized, open-label, phase II study exploring the role of molecular features in treating ALKpositive patients. A total of 116 stage III/IV NSCLC patients will be randomized to receive first-line treatment with brigatinib or with other second-generation ALK inhibitors. Tumor tissue will be collected at the baseline, while blood samples at the baseline and with every radiologic assessment. All the patients will receive a second-line treatment with

Table 2 Ongoing Phase II-III Clinical Trials with Brigatinib in Advanced ALK-Positive NSCLC

\begin{tabular}{|c|c|c|c|c|c|}
\hline Trial & $\begin{array}{l}\text { Phase } \\
\text { Trial }\end{array}$ & $\begin{array}{l}\text { Estimated } \\
\text { Enrollment }\end{array}$ & Main Inclusion Criteria & Arms & $\begin{array}{l}\text { Primary } \\
\text { Endpoint }\end{array}$ \\
\hline $\begin{array}{l}\text { NCT043I8938 (ABP } \\
\text { trial) }\end{array}$ & II & 116 pts & $\begin{array}{l}\text { No prior therapy for advanced ALK- } \\
\text { positive NSCLC }\end{array}$ & $\begin{array}{l}\text { Brigatinib vs second- } \\
\text { generation ALK inhibitor }\end{array}$ & PFS* \\
\hline NCT03596866 (ALTA-3) & III & 246 pts & $\begin{array}{l}\text { PD while on crizotinib no other ALK } \\
\text { inhibitor other than crizotinib }\end{array}$ & Brigatinib vs alectinib & PFS \\
\hline $\begin{array}{l}\text { NCT02706626 (ATOMIC } \\
\text { ARI-AT-002 trial) }\end{array}$ & II & $120 \mathrm{pts}$ & $\begin{array}{l}\text { Previous treatment with a second- } \\
\text { generation ALK inhibitor }\end{array}$ & Brigatinib & ORR \\
\hline NCT04074993 & II & 35 pts & $\begin{array}{l}\text { Previous treatment with one ALK inhibitor } \\
\text { ALK-rearrangement, as detected via the } \\
\text { blood somatic mutation assay }\end{array}$ & Brigatinib & ORR \\
\hline NCT04223596 (CUBIK) & II & 33 pts & No prior treatment for advanced NSCLC & Brigatinib & ORR \\
\hline NCT04634IIO (B3i trial) & II & 19 pts & Previously untreated brain metastases & Brigatinib & DCR \\
\hline
\end{tabular}

Note: *Of first-line treatment.

Abbreviations: Pts, patients; PFS, progression-free survival; NSCLC, non-small cell lung cancer; ORR, objective response rate; DCR, disease control rate. 
any other ALK inhibitor at the time of progression. Investigator's choice will be based on the next-generation sequencing (NGS)-based molecular tumor profile of tissue rebiopsies and liquid biopsies. ${ }^{36}$

The ALTA-3 trial is a Phase III study designed to compare the efficacy and safety of brigatinib vs alectinib in locally advanced or metastatic ALK-positive NSCLC patients who have progressed on crizotinib.

Patients who have previously received an ALK inhibitor other than crizotinib or more than two lines of systemic therapy (other than crizotinib) are excluded. In this study, approximately 246 patients will be enrolled. The primary endpoint is PFS assessed by BIRC. ${ }^{37}$

In the two-stage Simon design, phase II ATOMIC ARI-AT-002 trial, brigatinib is evaluated in patients who have progressed on second-generation ALK-inhibitors. NGS analysis of pretreatment tumor samples and circulating tumor DNA (ctDNA) to study $A L K$ resistance mutations and other resistance mechanisms are performed. The primary endpoint of the study is ORR. If greater than or equal to two responses were achieved among 20 eligible patients in the first stage, 20 additional patients would be accrued in stage 2. Results of the interim analysis, performed after the enrolment of 20 patients, have been published. Brigatinib was demonstrated to be active in this subset of patients with an ORR of $40 \%{ }^{38}$

A phase II study evaluating the efficacy of brigatinib in patients with inoperable stage III or metastatic NSCLC progressed on one previous ALK inhibitor, harboring $A L K$ rearrangement detected via the blood somatic mutation assay is ongoing. ${ }^{39}$

Furthermore, the clinical utility of liquid biopsy as a tool to assess the evolution of brigatinib treated ALK-positive NSCLC patients is under investigation in the phase 2 CUBIK study. ${ }^{40}$

The B3i trial is a single-arm, phase II study to evaluate first-line treatment with brigatinib in ALK-positive NSCLC patients with brain metastases not previously irradiated. If brigatinib demonstrates high intracranial disease control rates, this study could support first-line treatment with brigatinib for carefully selected patients with brain metastases. ${ }^{41}$

\section{Conclusions}

The FDA has approved five ALK inhibitors (alectinib, brigatinib, ceritinib, lorlatinib, and crizotinib) for the first-line treatment of ALK-positive advanced NSCLC. Recently, also ensartinib, a novel second-generation ALK inhibitor, was demonstrated to be an effective option in the first-line setting, showing significant longer PFS (25.8 months vs 12.7 months, HR, $0.51, \mathrm{p}<0.001)$ and a higher rate of intracranial response $(63.6 \%$ vs $21.1 \%)$ than crizotinib in phase III eXalt3 study. ${ }^{42}$ However, the optimal treatment sequence of ALK inhibitors remains a matter to debate.

Brigatinib showed clinical activity in treatment-naïve patients and in patients who have progressed on crizotinib. Furthermore, brigatinib is generally well-tolerated thanks to its manageable safety profile. A small subset of patients experienced EOPEs; however, step-up dosing can reduce the risk of developing pulmonary toxicity.

To date, no head-to-head randomized clinical trials comparing brigatinib with alectinib have been conducted in a firstline setting.

Results from an indirect treatment comparison showed no significant difference in terms of PFS between brigatinib and alectinib. However, in the subset of patients with brain metastases, brigatinib demonstrated greater efficacy than alectinib. The peculiar structural features of brigatinib could explain this finding. Indeed, brigatinib has a dimetylphosphine oxide (DMPO) that confers high water solubility, low lipophilicity, and low protein-binding to the drug, resulting in more significant activity in the brain metastases. ${ }^{43}$

The J-ALTA trial and preliminary results of the phase II ATOMIC ARI-AT-002 trial supported the administration of brigatinib after second-generation ALK inhibitor. Notably, brigatinib demonstrated activity similar to lorlatinib but with a more favorable safety profile in the post-next-generation ALK inhibitor setting. ${ }^{44}$

The optimal treatment for overcoming the resistance mechanisms acquired after progression with brigatinib is unknown. To date, no large-scale analysis of resistance mechanisms with brigatinib therapy has been published. Results from the multicentric real-world study evaluating brigatinib in ALK-positive advanced pre-treated NSCLC (BrigALK2 study) demonstrated the activity of subsequent ALK inhibitors after brigatinib. The median duration of lorlatinib post crizotinib was 5.3 months, and the median OS from lorlatinib initiation was 14.1 months. ${ }^{45}$

To date, tissue or liquid biopsies performed at the time of disease progression, even though not mandated by the approval status of any ALK inhibitor, could be very helpful for the management of ALK-positive NSCLC patients. 
Further studies are still required to establish sequencing recommendations.

\section{Disclosure}

Dr Erika Rijavec reports personal fees from BRISTOL-MYERS SQUIBB, outside the submitted work. Professor Francesco Grossi reports grants, personal fees from Bristol Myers Squibb, personal fees from Merck Sharp and Dome, personal fees from Eli-Lilly, personal fees from Amgen, personal fees from Sanofi, personal fees from Novartis, personal fees from Astra Zeneca, personal fees from Roche, personal fees from Pierre Fabre, personal fees from Celgene, personal fees from Takeda, personal fees from Boehringer, personal fees from Merck, during the conduct of the study. Dr Carlo Genova reports personal fees from Astra Zeneca, personal fees from Boehringer Ingelheim, grants from Bristol Myers Squibb, personal fees from Merck Sharp \& Dohme, personal fees from Roche, personal fees from Takeda, personal fees from ThermoFisher, grants from Italian Ministry of Health, outside the submitted work. The authors report no other conflicts of interest in this work.

\section{References}

1. Sung H, Ferlay J, Siegel RL, et al. Global cancer statistics 2020: GLOBOCAN estimates of incidence and mortality worldwide for 36 cancers in 185 countries. CA Cancer J Clin. 2021;71(3):209-249. doi:10.3322/caac.21660

2. Soda M, Choi YL, Enomoto M, et al. Identification of the transforming EML4-ALK fusion gene in non-small-cell lung cancer. Nature. 2007;448 (7153):561-566. doi:10.1038/nature05945

3. McKeage MJ, Tin Tin S, Khwaounjoo P, et al. Screening for anaplastic lymphoma kinase (ALK) gene rearrangements in non-small-cell lung cancer in New Zealand. Intern Med J. 2020;50(6):716-725. doi:10.1111/imj.14435

4. Colombino M, Paliogiannis P, Cossu A, et al. EGFR, KRAS, BRAF, ALK, and cMET genetic alterations in 1440 Sardinian patients with lung adenocarcinoma. BMC Pulm Med. 2019;19(1):209. doi:10.1186/s12890-019-0964-x

5. Shaw AT, Yeap BY, Mino-Kenudson M, et al. Clinical features and outcome of patients with non-small-cell lung cancer who harbor EML4-ALK. $J$ Clin Oncol. 2009;27(26):4247-4253. doi:10.1200/JCO.2009.22.6993

6. Schrank Z, Chhabra G, Lin L, et al. Current molecular-targeted therapies in NSCLC and their mechanism of resistance. Cancers. 2018;10(7):224. doi:10.3390/cancers10070224

7. Kazandjian D, Blumenthal GM, Chen HY, et al. FDA approval summary: crizotinib for the treatment of metastatic non-small cell lung cancer with anaplastic lymphoma kinase rearrangements. Oncologist. 2014;19(10):e5-e11. doi:10.1634/theoncologist.2014-0241

8. Huang WS, Liu S, Zou D, et al. Discovery of brigatinib (AP26113), a phosphine oxide-containing, potent, orally active inhibitor of anaplastic lymphoma kinase. $J$ Med Chem. 2016;59(10):4948-4964. doi:10.1021/acs.jmedchem.6b00306

9. Tugnait M, Gupta N, Hanley MJ, et al. Effects of strong CYP2C8 or CYP3A inhibition and CYP3A induction on the pharmacokinetics of brigatinib, an oral anaplastic lymphoma kinase inhibitor, in healthy volunteers. Clin Pharmacol Drug Dev. 2020;9(2):214-223. doi:10.1002/ cpdd.723

10. Tugnait M, Gupta N, Hanley MJ, et al. The effect of a high-fat meal on the pharmacokinetics of brigatinib, an oral anaplastic lymphoma kinase inhibitor, in healthy volunteers. Clin Pharmacol Drug Dev. 2019;8(6):734-741. doi:10.1002/cpdd.641

11. Zhang S, Anjum R, Squillace R, et al. The potent ALK inhibitor brigatinib (AP26113) overcomes mechanisms of resistance to first- and secondgeneration ALK inhibitors in preclinical models. Clin Cancer Res. 2016;22(22):5527-5538. doi:10.1158/1078-0432.CCR-16-0569

12. Horn L, Whisenant JG, Wakelee H, et al. Monitoring therapeutic response and resistance: analysis of circulating tumor DNA in patients with ALK+ lung cancer. $J$ Thorac Oncol. 2019;14(11):1901-1911. doi:10.1016/j.jtho.2019.08.003

13. Gettinger SN, Bazhenova LA, Langer CJ, et al. Activity and safety of brigatinib in ALK-rearranged non-small-cell lung cancer and other malignancies: a single-arm, open-label, phase 1/2 trial. Lancet Oncol. 2016;17(12):1683-1696. doi:10.1016/S1470-2045(16)30392-8

14. Kim DW, Tiseo M, Ahn MJ, et al. Brigatinib in patients with crizotinib-refractory anaplastic lymphoma kinase-positive non-small-cell lung cancer: a randomized, multicenter phase II trial. J Clin Oncol. 2017;35(22):2490-2498. doi:10.1200/JCO.2016.71.5904

15. U.S. Food \& Drug Administration. Brigatinib; [updated July 25, 2017]. Available from: https://www.fda.gov/drugs/resources-informationapproved-drugs/brigatinib. Accessed November 20, 2021.

16. Camidge DR, Kim DW, Tiseo M, et al. Exploratory analysis of brigatinib activity in patients with anaplastic lymphoma kinase-positive non-smallcell lung cancer and brain metastases in two clinical trials. J Clin Oncol. 2018;36(26):2693-2701. doi:10.1200/JCO.2017.77.5841

17. Huber RM, Hansen KH, Paz-Ares Rodríguez L, et al. Brigatinib in crizotinib-refractory ALK+ NSCLC: 2-year follow-up on systemic and intracranial outcomes in the phase 2 ALTA trial. $J$ Thorac Oncol. 2020;15(3):404-415. doi:10.1016/j.jtho.2019.11.004

18. Shaw AT, Solomon BJ, Besse B, et al. $A L K$ resistance mutations and efficacy of lorlatinib in advanced anaplastic lymphoma kinase-positive nonsmall-cell lung cancer. $J$ Clin Oncol. 2019;37(16):1370-1379. doi:10.1200/JCO.18.02236

19. Shaw AT, Kim TM, Crinò L, et al. Ceritinib versus chemotherapy in patients with ALK-rearranged non-small-cell lung cancer previously given chemotherapy and crizotinib (ASCEND-5): a randomised, controlled, open-label, phase 3 trial. Lancet Oncol. 2017;18(7):874-886. doi:10.1016/ S1470-2045(17)30339-X

20. Shaw AT, Gandhi L, Gadgeel S, et al. Alectinib in ALK-positive, crizotinib-resistant, non-small-cell lung cancer: a single-group, multicentre, phase 2 trial. Lancet Oncol. 2016;17(2):234-242. doi:10.1016/S1470-2045(15)00488-X

21. Lenderking WR, Lin H, Speck RM, et al. Patient-reported outcomes and quality of life in advanced ALK+ non-small-cell lung cancer trial of brigatinib (ALTA). Future Oncol. 2019;15(24):2841-2855. doi:10.2217/fon-2019-0185

22. Kawata AK, Lenderking WR, Eseyin OR, et al. Converting EORTC QLQ-C30 scores to utility scores in the brigatinib ALTA study. $J$ Med Econ. 2019;22(9):924-935. doi:10.1080/13696998.2019.1624080 
23. Gettinger SN, Huber RM, Kim DW, et al. Brigatinib in ALK+ crizotinib-refractory non-small cell lung cancer: final results of the phase $1 / 2$ and phase 2 (ALTA) trials. Presented at the 2021 ASCO Annual Meeting; June 4-8; Virtual Abstract 9071; 2021.

24. Camidge DR, Kim HR, Ahn MJ, et al. Brigatinib versus crizotinib in ALK-positive non-small-cell lung cancer. N Engl J Med. 2018;379(21):20272039. doi:10.1056/NEJMoa1810171

25. Camidge DR, Niu H, Kim HR, et al. Correlation of baseline molecular and clinical variables with ALK inhibitor efficacy in ALTA-1L. J Clin Oncol. 2020;38(15):9517. doi:10.1200/JCO.2020.38.15_suppl.9517

26. Christopoulos P, Kirchner M, Endris V, Stenzinger A, Thomas M. EML4-ALK V3, treatment resistance, and survival: refining the diagnosis of ALK $^{+}$NSCLC. $J$ Thorac Dis. 2018;10(17):S1989-S1991. doi:10.21037/jtd.2018.05.61

27. Kron A, Alidousty C, Scheffler M, et al. Impact of TP53 mutation status on systemic treatment outcome in ALK-rearranged non-small-cell lung cancer. Ann Oncol. 2018;29(10):2068-2075. doi:10.1093/annonc/mdy333

28. Christopoulos $\mathrm{P}$, Kirchner M, Bozorgmehr F, et al. Identification of a highly lethal $\mathrm{V}^{+} \mathrm{TP}^{2} 3^{+}$subset in $\mathrm{ALK}^{+}$lung adenocarcinoma. Int $J$ Cancer. 2019;144(1):190-199. doi:10.1002/ijc.31893

29. Camidge DR, Kim HR, Ahn MJ, et al. Brigatinib versus crizotinib in advanced ALK inhibitor-naive ALK-positive non-small cell lung cancer: second interim analysis of the phase III ALTA-1L trial. J Clin Oncol. 2020;38(31):3592-3603. doi:10.1200/JCO.20.00505

30. Garcia Campelo MR, Lin HM, Zhu Y, et al. Health-related quality of life in the randomized phase III trial of brigatinib vs crizotinib in advanced ALK inhibitor-naive ALK + non-small cell lung cancer (ALTA-1L). Lung Cancer. 2021;155:68-77. doi:10.1016/j.lungcan.2021.03.005

31. U.S. Food \& Drug Administration. FDA approves brigatinib for ALK-positive metastatic NSCLC; [updated May 26, 2020]. Available from: https:// www.fda.gov/drugs/resources-information-approved-drugs/fda-approves-brigatinib-alk-positive-metastatic-nsclc. Accessed November $20,2021$.

32. Camidge DR, Kim HR, Ahn MJ, et al. Brigatinib versus crizotinib in ALK inhibitor-naive advanced ALK-positive NSCLC: final results of phase 3 ALTA-1L trial. $J$ Thorac Oncol. 2021;16(12):2091-2108. doi:10.1016/j.jtho.2021.07.035

33. Nishio M, Yoshida T, Kumagai T, et al. Brigatinib in Japanese patients with ALK-positive NSCLC previously treated with alectinib and other tyrosine kinase inhibitors: outcomes of the phase 2 J-ALTA trial. $J$ Thorac Oncol. 2021;16(3):452-463. doi:10.1016/j.jtho.2020.11.004

34. Kondo M, Sugawara S, Yokoyama T, et al. Brigatinib in Japanese patients with anaplastic lymphoma kinase (ALK)-positive non-small cell lung cancer (NSCLC): first results from the J-ALTA tyrosine kinase inhibitor (TKI)-naive expansion cohort. Presented at the 2021 ASCO Annual Meeting; June 4-8; Virtual Abstract 9042; 2021.

35. Kim ES, Barlesi F, Mok T, et al. ALTA-2: phase II study of brigatinib in patients with ALK-positive, advanced non-small-cell lung cancer who progressed on alectinib or ceritinib. Future Oncol. 2021;17(14):1709-1719. doi:10.2217/fon-2020-1119

36. Christopoulos P, Bozorgmehr F, Brückner L, et al. Brigatinib versus other second-generation ALK inhibitors as initial treatment of anaplastic lymphoma kinase positive non-small cell lung cancer with deep phenotyping: study protocol of the ABP trial. BMC Cancer. 2021;21(1):743. doi:10.1186/s12885-021-08460-w

37. Popat S, Liu G, Lu S, Song G, Ma X, Yang JC. Brigatinib vs alectinib in crizotinib-resistant advanced anaplastic lymphoma kinase-positive nonsmall-cell lung cancer (ALTA-3). Future Oncol. 2021;17(32):4237-4247. doi:10.2217/fon-2021-0608

38. Stinchcombe TE, Doebele RC, Wang X, Gerber DE, Horn L, Camidge DR. Preliminary clinical and molecular analysis results from a single-arm phase 2 trial of brigatinib in patients with disease progression after next-generation ALK tyrosine kinase inhibitors in advanced ALK+ NSCLC. $J$ Thorac Oncol. 2021;16(1):156-161. doi:10.1016/j.jtho.2020.09.018

39. ClinicalTrials.gov. Brigatinib in ALK-positive NSCLC identified via blood-based assays; [updated September 10, 2020]. Available from: https:// clinicaltrials.gov/ct2/show/NCT04074993?term=nsclc\&cond=brigatinib\&phase=123\&draw=2\&rank=4. Accessed November $20,2021$.

40. ClinicalTrials.gov. Clinical utility of liquid biopsy in brigatinib ALK+ patients (CUBIK); [updated August 11, 2021]. Available from: https:// clinicaltrials.gov/ct2/show/NCT04223596?term=nsclc\&cond=brigatinib\&phase=123\&draw=2\&rank=10. Accessed November $20,2021$.

41. ClinicalTrials.gov. Brigatinib before brain irradiation trial (B3i Trial); [updated March 22, 2021]. Available from: https:/clinicaltrials.gov/ct2/show/ NCT04634110?term=nsclc\&cond=brigatinib\&phase=123\&draw=2\&rank=9. Accessed November 20, 2021.

42. Horn L, Wang Z, Wu G, et al. Ensartinib vs crizotinib for patients with anaplastic lymphoma kinase-positive non-small cell lung cancer: a randomized clinical trial. JAMA Oncol. 2021;7(11):1617-1625. doi:10.1001/jamaoncol.2021.3523

43. Ando K, Manabe R, Kishino Y, et al. Comparative efficacy and safety of lorlatinib and alectinib for ALK-rearrangement positive advanced nonsmall cell lung cancer in Asian and non-Asian patients: a systematic review and network meta-analysis. Cancers. 2021;13(15):3704. doi:10.3390/ cancers 13153704

44. Solomon BJ, Besse B, Bauer TM, et al. Lorlatinib in patients with ALK-positive non-small-cell lung cancer: results from a global phase 2 study. Lancet Oncol. 2018;19(12):1654-1667. doi:10.1016/S1470-2045(18)30649-1

45. Descourt R, Pérol M, Rousseau-Bussac G, et al. BrigALK2 study: a multicentric real-world study evaluating brigatinib in ALK positive advanced pre-treated non-small cell lung cancers: long-term follow-up with focus on lorlatinib efficacy after brigatinib. Presented at the 2021 ASCO Annual Meeting; June 4 -8; Virtual Abstract 1200P; 2021.

Clinical Pharmacology: Advances and Applications

Dovepress

Publish your work in this journal

Clinical Pharmacology: Advances and Applications is an international, peer-reviewed, open access journal publishing original research, reports, reviews and commentaries on all areas of drug experience in humans. The manuscript management system is completely online and includes a very quick and fair peer-review system, which is all easy to use. Visit http://www.dovepress.com/testimonials.php to read real quotes from published authors.

Submit your manuscript here: https://www.dovepress.com/clinical-pharmacology-advances-and-applications-journal 